\title{
DYNAMICS OF EXTERNAL OCULAR BLOOD FLOW STUDIED BY SCANNING ANGIOGRAPHIC MICROSCOPY
}

\author{
L. DAVID ORMEROD, ENRIQUE FARIZA and ROBERT H. WEBB \\ Boston, Massachusetts
}

\begin{abstract}
SUMMARY
The scanning angiographic microscope (SAM) provides a solution to the considerable technical difficulties associated with conventional episcleral fluorescein angiography. Standardised anterior segment fluorescein videoangiograms were performed using the SAM in each episcleral quadrant of the right eye in 6 normal subjects; frame-by-frame analysis proved important. Centripetal flow was seen in all 37 scleral perforating arteries investigated. Other features were the marked individual variability, much larger vertical anterior ciliary arteries, the high frequency of arteriovenous anastomoses, the complex flow patterns, the absence of a 'watershed' zone between anterior ciliary and posterior episcleral circulations, a characteristic and discontinuous distribution of 'leaky' episcleral veins, and the primacy of venous drainage into the plexus of muscular veins. Reports of retrograde blood flow in the anterior ciliary arteries in most fluorescein angiographic studies are probably incorrect, the result of unappreciated methodological problems. The SAM is an important advance on previous anterior segment fluorescein angiography techniques.
\end{abstract}

The blood supply to the anterior segment ${ }^{1-3}$ is derived from two circulations, one external to the eye and one with an intraocular route. The external circulation comprises the anterior ciliary arteries, which are continuations of the muscular arteries of the rectus muscles. The anterior ciliary arteries emerge from the rectus muscles posterior to the transition from muscle to tendon, ${ }^{4}$ and run forward along the tendon before dividing within the episcleral tissues into branches ${ }^{5}$ that supply an extensive

From: Schepens Eye Research Institute, Department of Ophthalmology, Harvard Medical School, Boston, Massachusetts, USA.

Correspondence to: L. David Ormerod, FRCOphth, MRCP, Department of Ophthalmology, University of South Florida, Tampa, FL 33612-4799, USA. circumepiscleral plexus anterior to the rectus muscle insertions. A few large branches of the anterior ciliary arteries pass through scleral channels into the eye, where they arborise almost immediately to supply the iris, intramuscular circle, ciliary muscle, and anterior choriocapillaris. ${ }^{5,6}$

The intraocular component of the anterior segment blood supply ${ }^{6-8}$ consists of the medial and lateral long posterior ciliary arteries, which are the main source for the major arterial circle of the iris; they also supply the ciliary processes, and give branches to the ciliary muscle. A primate study has suggested that an extensive collateral system may occur in the intramuscular circle between the anterior ciliary and long posterior ciliary circulations, ${ }^{8}$ although this has been disputed in two human studies. ${ }^{6,7}$

It is generally accepted that the anterior ciliary arteries provide a major part of the vascular supply to the anterior uvea via their scleral perforating branches. $^{1-3}$ This view is supported by the development of anterior segment ischaemia following tenotomy of the vertical rectus muscles, particularly when combined with additional tenotomies, ${ }^{4,9-11}$ and by the results of studies involving corrosion vascular castings, ${ }^{5-8}$ the injection of labelled microspheres, ${ }^{12,13}$ and extraocular muscle tenotomies in primates. $^{14-16}$

However, several investigations of the circulatory dynamics using segment fluorescein angiography have concluded that blood flow in the anterior ciliary arteries is principally centrifugal or retrograde in direction, ${ }^{17-25}$ and that the anterior ocular surface is predominantly supplied from within the globe. These angiographic observations appear to be at odds with most of the non-angiographic clinical and experimental data. Conventional angiographic studies use off-axis light sources (to reduce highlights) that produce markedly uneven illumination 
across the curved episcleral surface. The resulting variable fluorescence excitation may be exacerbated by differences in scleral reflectance at increasingly acute angles of incidence of excitation light. Furthermore, most studies have the scleral perforating vessels in the central, highly illuminated parts of the field and the anterior ciliary arteries in the less illuminated periphery. The interpretation of photographic records is restricted further by their dependence upon slow flash-recycle times and the highly non-linear characteristics of photographic film at low light intensities. The low light levels that are a hallmark of a fluorescent light source also lead to a shallow depth of field when using conventional photographic or video close-up cameras, with the periphery of the field commonly out of focus. Finally, light reflexes from the tear film and 'pseudofluorescence' commonly interfere with angiography.

These problems are controlled by the configuration of the scanning angiographic microscope (SAM) ${ }^{26}$ a recent development of the scanning laser ophthalmoscope. ${ }^{27,28}$ The field is co-axially scanned by a 488-nm laser (Siemens, Munich, Germany) raster with a long depth of focus. ${ }^{26}$ Tight filtering provides a dark (scleral) background without light reflexes. The returned light is collected by a 1-mm avalanche photodiode detector and integral amplifier (RCA, Vaudreinline, Quebec, Canada), a highly sensitive detection system with high signal-tonoise ratio. Real-time TV operation and S/VHS video recording are other important advantages.

The scanning functions of the SAM are tightly integrated electronically with the TV monitor image signal, resulting in point-to-point reciprocity. ${ }^{29}$ Each pixel of the monitor corresponds to a particular point in the illumination raster. A modest limitation of image resolution compared with conventional photography results from restrictions inherent in a 500-line TV raster. No useful information appears to be lost in SAM anterior segment videoangiography, and there is greatly improved image uniformity across the field. ${ }^{26}$ The recorded S/VHS video image is a little degraded compared with the 'live' image on the monitor. ${ }^{26}$ The video frame rate of $30 \mathrm{~Hz}$ provides 30 frames (60 fields) per second, rather than the one field every few seconds in conventional photographic angiography. The analysis of SAM video images is a dynamic and not a static process, but, for purposes of publication, images are obtained by direct photography of the monitor screen, collecting approximately seven video frames over an 0.25 -second exposure. $^{26}$ These photographed images are significantly inferior to the video image.

In view of the conflicting opinions regarding the vascular dynamics of the anterior ciliary and episcleral vasculature, we have systematically stu- died the transit of fluorescein in normal human subjects using this new technology.

\section{MATERIALS AND METHODS}

We performed anterior segment fluorescein videoangiograms in each of the episcleral quadrants of the right eye in 6 normal human volunteers ( 4 men, 2 women; age range $21-42$ years). The individuals had no ocular or systemic disease and were not taking medication. The results of ocular examination were normal in each subject. Colour photographs of all four quadrants aided interpretation of the videoangiography. The protocol followed the tenets of the Helsinki Declaration and was approved by the institutional human experimentation committee. All subjects gave their informed consent.

The methodology has been described previously. ${ }^{26}$ Briefly, $2 \mathrm{ml}$ of $25 \%$ sodium fluorescein was injected rapidly into an antecubital vein via a three-way tap during a sustained period of inspiration. The cannula was flushed immediately with a 5-ml bolus of balanced salt solution coincidental with a rapid expiration. ${ }^{30}$ Video recording with an audio hookup was begun before fluorescein injection and continued for 2-3 minutes.

The four quadrants were studied at four separate sessions. Standard visual fixation points were provided by red, light-emitting diodes mounted at visual angles of $42^{\circ}$ on either side of the midline from the neutral eye position, and at $30^{\circ}$ elevation and $45^{\circ}$ depression in the midline. Insertion of a lid speculum after topical proparacaine $0.5 \%$ anaesthesia was necessary for the superior quadrant angiograms. Movement of the headrest during the fluorescein transit permitted extension of the fields into the fornices.

The scanning angiographic microscope was configured to give a $10 \times 10 \mathrm{~mm}$ field and $\times 25$ magnification at the TV monitor. Scleral irradiance of the scanned 488-nm blue laser was standardised at $100-110 \mu \mathrm{W} / \mathrm{cm}^{2}$. A diagram of the SAM is shown in Fig. 1. Because the tight filtration in the collection optics provided a black background, a second 'focusing and alignment' channel using a blue reflector dichroic beam splitter and separate monitor was provided to image the episclera in unfiltered blue light. The instrumentation of the SAM has been discussed in detail elsewhere. ${ }^{26}$ The fluorescein angiograms were recorded on S/VHS videotape using a Panasonic (Secaucus, NJ, USA) S/VHS AG-7300 video recording unit.

Analysis of the fluorescein transits was undertaken according to a detailed protocol. The angiographic sequence was timed from the completion of fluorescein injection. Interpretation of the video images was enhanced by the frame-by-frame advance and reverse playback features of the 


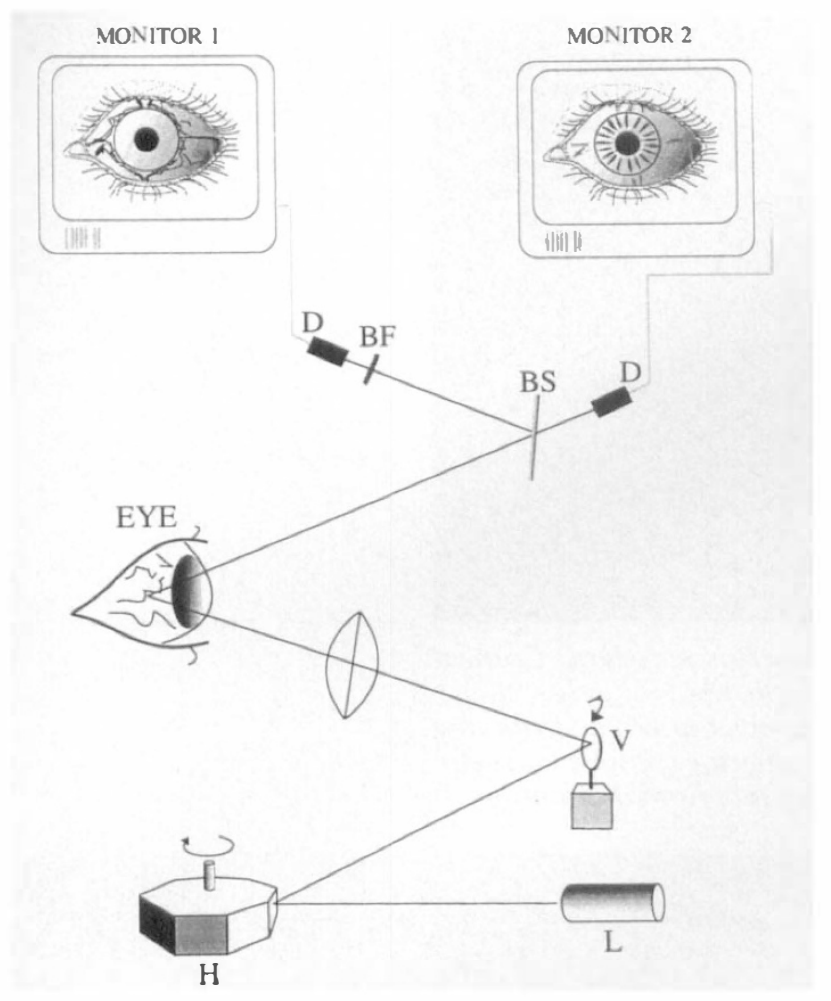

Fig. 1. Diagrammatic representation of the scanning angiographic microsope (SAM). The incident and collection optics are the same, although not shown diagrammatically. The system is not truly confocal - a large $(10 \mathrm{~mm})$ aperture is placed at a conjunctival conjugate to optimise fluorescence intensity. L, $488 \mathrm{~nm}$ argon ion blue laser light source; $H$, 25-faceted rotating polygon mirror spinning at $37800 \mathrm{rpm}$; $V$, large vertical $60 \mathrm{~Hz}$ galvanometer mirror; an aspheric 28-dioptre lens focuses the illumination raster on the episclera; BS, dichroic beam splitter; BF, Schott Y52 long-wave-pass barrier filter $(520 \mathrm{~nm}) ; \mathrm{D}, 1 \mathrm{~mm}$ avalanche photodiode detector with integral amplifier.

Panasonic video unit and by use of a high-resolution monitor. For purposes of illustration, a photographic record was made directly from the videoscreen using a Nikon F2 camera, Nikkor 50-mm lens (Nikon, Garden City, NY, USA) and Kodak Plus X Pan film (ASA 125: Eastman Kodak, Rochester, NY, USA) with an aperture of $\mathrm{f} 4$ and an exposure of 0.25 second. The vascular anatomy revealed at videoangiography was compared with slit lamp examination and four-quadrant colour photography using Ektachrome film (Kodak, Rochester, NY, USA).

\section{RESULTS}

The episcleral vasculature is an unusually complex vascular bed characterised by multiple overlying vascular layers. Fluorescein transit is rapid, angiographic staging is imprecise, and vascular detail is soon compromised by extensive fluorescein leakage. Therefore, useful anatomical and clinical information is compressed into the first 30 seconds or so. Realtime interpretation of a videoangiographic record with frame-by-frame playback facilities in this context is invaluable. It should be remembered that the quality of our illustrations is much inferior to the original video images. The figures were taken by photographing the TV monitor, and seven video frames in dynamic sequence ( 0.25 -second exposure) were averaged.

\section{Antecubital-Episcleral Perfusion Time}

The time taken to first detect fluorescein in the anterior ciliary arteries was determined for each angiogram using standard injection conditions and the same injector (Table I). There was an approximate relationship between subject height and appearance time, but there was no significant difference by episcleral quadrant.

\section{Determination of Direction of Flow}

Careful observation of the fluorescent vascular images determined both highly fluorescent and darker spots moving throughout the episcleral vessels. These particles appeared and disappeared, but along every vessel, however small, they could be seen moving predominantly in a direction and at a rate consistent with the nature of that vessel. A small proportion of the particles appeared to move in the reverse direction, but this was believed to be due to the raster nature of the illuminating (and imaging) optics that periodically detected similar particles in a retrograde position as others tumbled away from the fluorescing surface of the blood column. Maximumcontrast and reduced-brightness settings on the monitor, slowing the videotape, and advance and reverse playback were useful in discerning the direction of flow. There was no directional movement seen in areas of venous leakage.

Table I. Antecubital-episcleral perfusion times (in seconds) of six normal subjects as assessed by episcleral quadrant

\begin{tabular}{|c|c|c|c|c|c|c|c|}
\hline \multirow[b]{2}{*}{ Subject } & \multirow[b]{2}{*}{ Sex } & \multirow{2}{*}{$\begin{array}{l}\text { Height } \\
\text { (ft, in) }\end{array}$} & \multicolumn{5}{|c|}{ Perfusion time in seconds by quadrant } \\
\hline & & & Temporal & Nasal & Inferior & Superior & Mean $( \pm S E)$ \\
\hline 1 & $\mathrm{~F}$ & $4^{\prime} 11 \frac{3 \prime \prime}{4}$ & 10 & 10 & 8 & 10 & $9.50( \pm 1.00)$ \\
\hline 2 & $\mathrm{~F}$ & $5^{\prime} 7 \frac{1}{2}^{\prime \prime}$ & 10 & 11 & 11 & 14 & $11.50( \pm 1.73)$ \\
\hline 3 & $\mathbf{M}$ & $5^{\prime} 7^{\prime \prime}$ & 16 & 15 & 13 & 17 & $15.25( \pm 1.71)$ \\
\hline 4 & $\mathbf{M}$ & $5^{\prime} 9 \frac{1}{2}^{\prime \prime}$ & 20 & 16 & 21 & 14 & $17.75( \pm 3.30)$ \\
\hline 5 & $\mathbf{M}$ & $5^{\prime} 10^{\frac{1}{2}}$ & 19 & 20 & 16 & 20 & $18.75( \pm 1.89)$ \\
\hline 6 & $\mathbf{M}$ & $5^{\prime} 11^{\prime \prime}$ & 16 & 19 & 23 & 18 & $19.00( \pm 2.94)$ \\
\hline
\end{tabular}

F, female; M, male. 


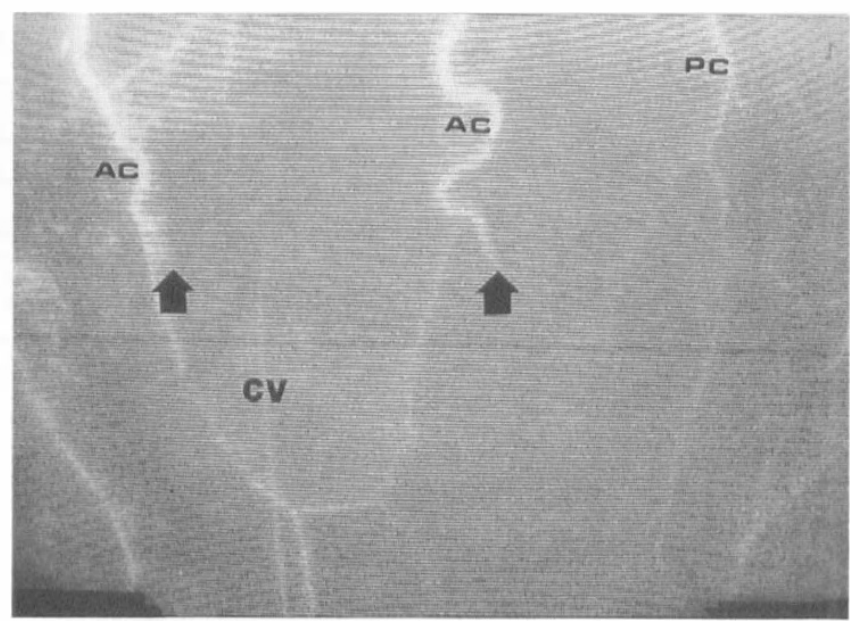

Fig. 2. Early arterial phase: superior episclera. Corneal limbus is at bottom of photograph. AC, anterior ciliary artery; $P C$, large posterior conjunctival artery contributing to anterior episcleral plexus; $C V$, anterior conjunctival vein; large arrow, perforating branch of anterior ciliary artery.

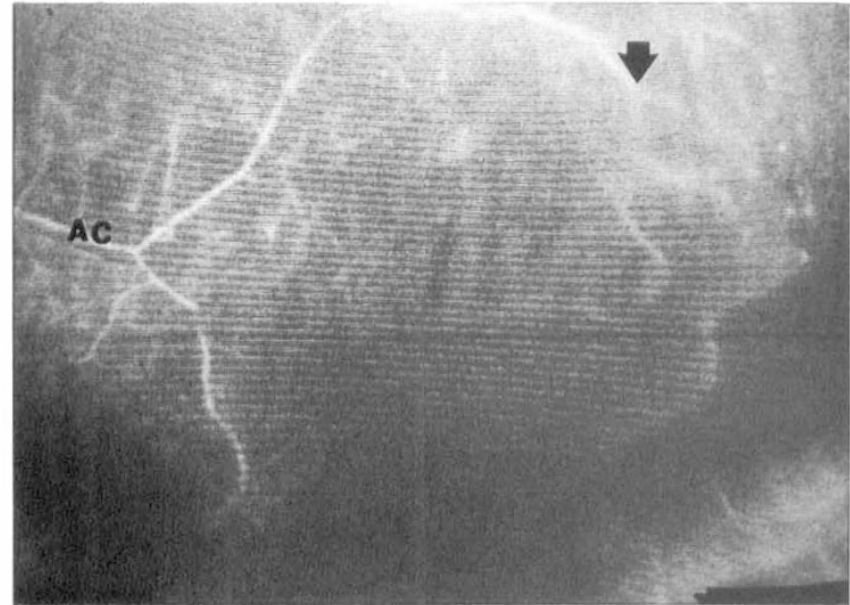

(a)

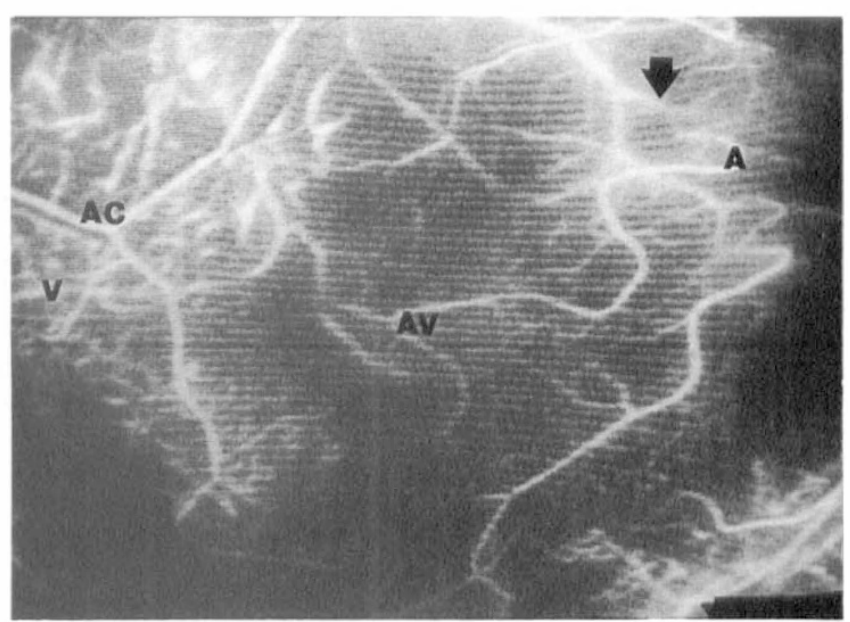

(b)

Fig. 3. Early (a) and late (b) arterial phase: temporal episclera. Corneal limbus is at right of photographs. AC, anterior ciliary artery; $A$, anterior episcleral artery; $A V$, arteriovenous anastomoses; $V$, episcleral vein; large arrow, perforating branch of anterior ciliary artery.

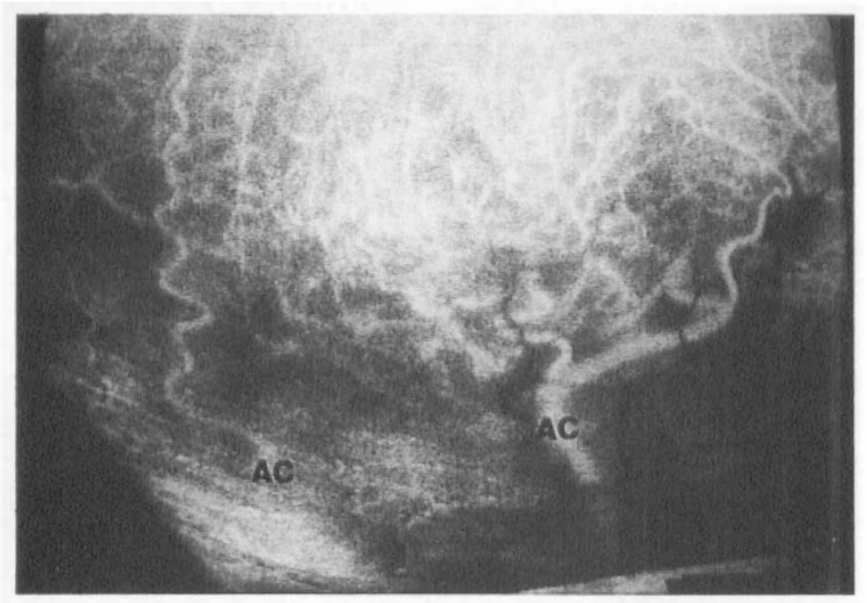

Fig. 4. Arteriovenous phase: inferior episclera. Corneal limbus is at top of photograph. AC, anterior ciliary artery. Note large temporal and nasal branches supplying anterior episcleral circle. Several overlying conjunctival veins are not filled. 


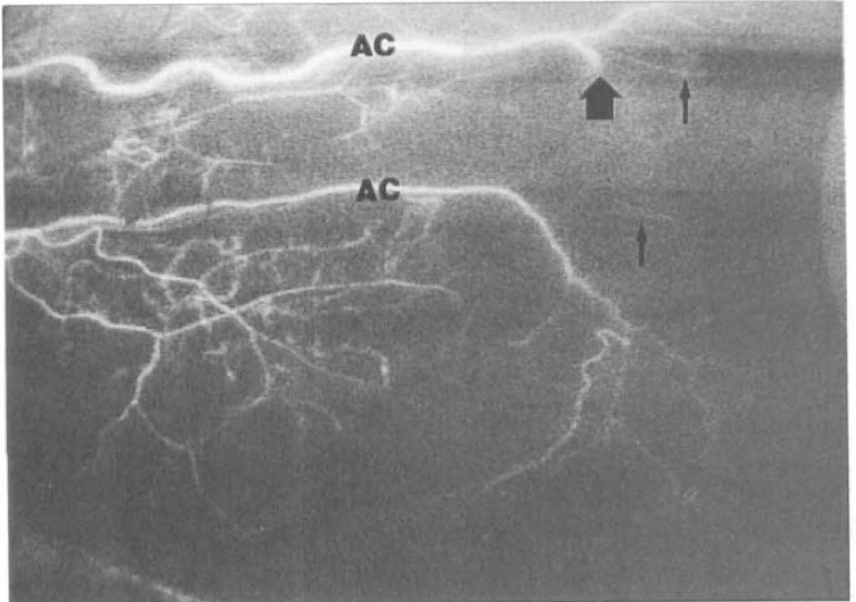

(a)

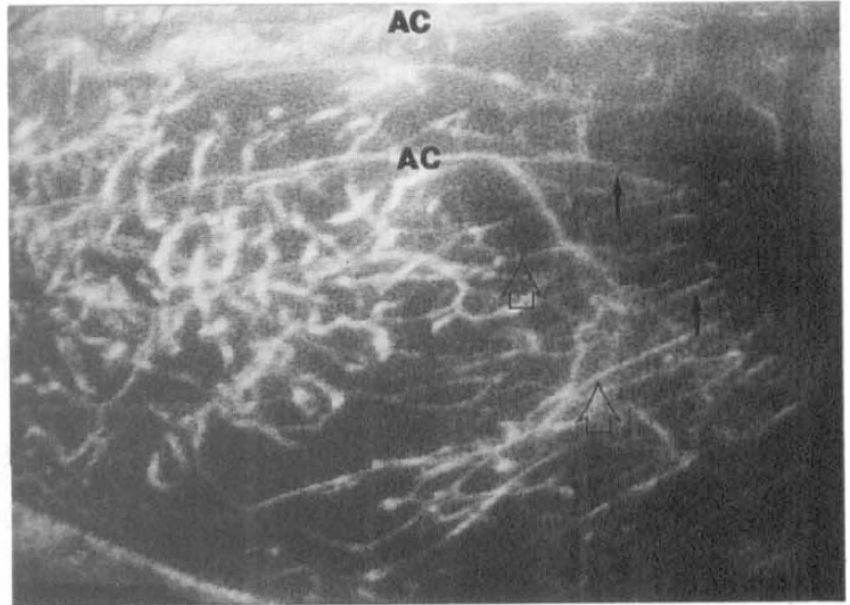

(b)

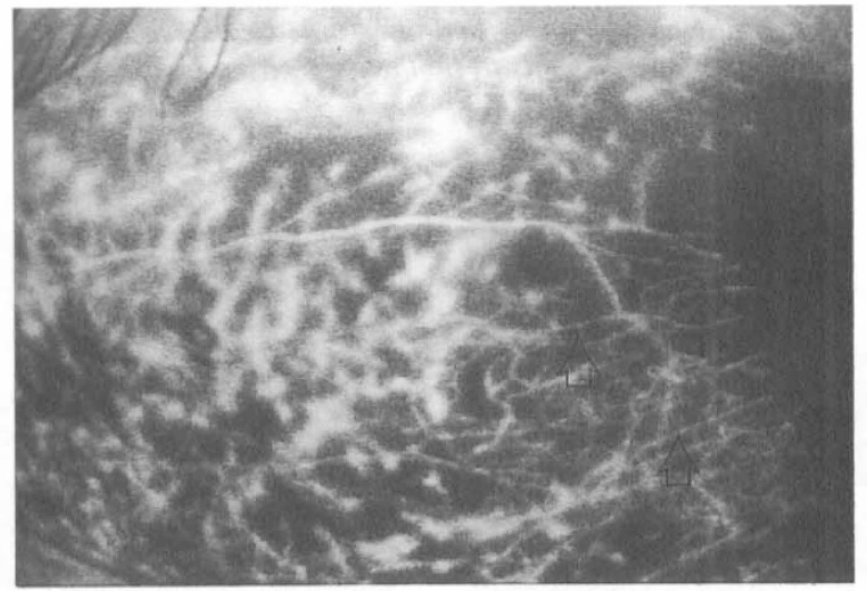

(c)

Fig. 5. Fluorescein transit: temporal episclera. Limbus is at right of photograph. (a) Midarterial phase. (b) Late arterial phase. (c) Arteriovenous phase. AC, anterior ciliary arteries; large arrow, perforating branch of anterior ciliary artery; small arrows, radial arterioles supplying limbus; open arrows, radial anterior conjunctival veins. Note characteristic site and character of fluorescein 'leakiness'.

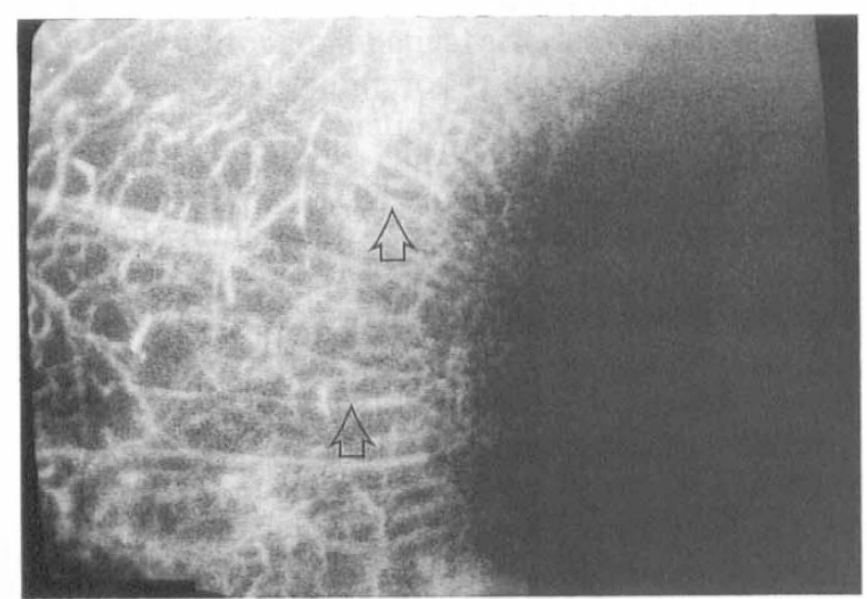

(a)

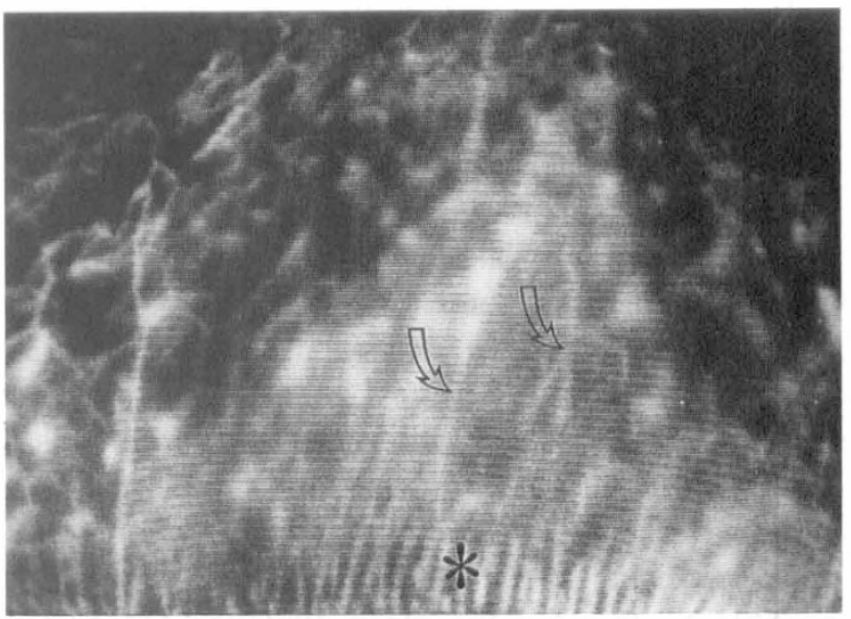

(b)

Fig. 6. Perilimbal vasculature: (a) Temporal. Shows small radial afferent arterioles, perilimbal arcades, and anterior episcleral circle. (b) Superior. Limbus is at lower border of photograph. Limbal palisades of Vogt (asterisk) are well seen. Open arrows, radial anterior conjunctival veins. 


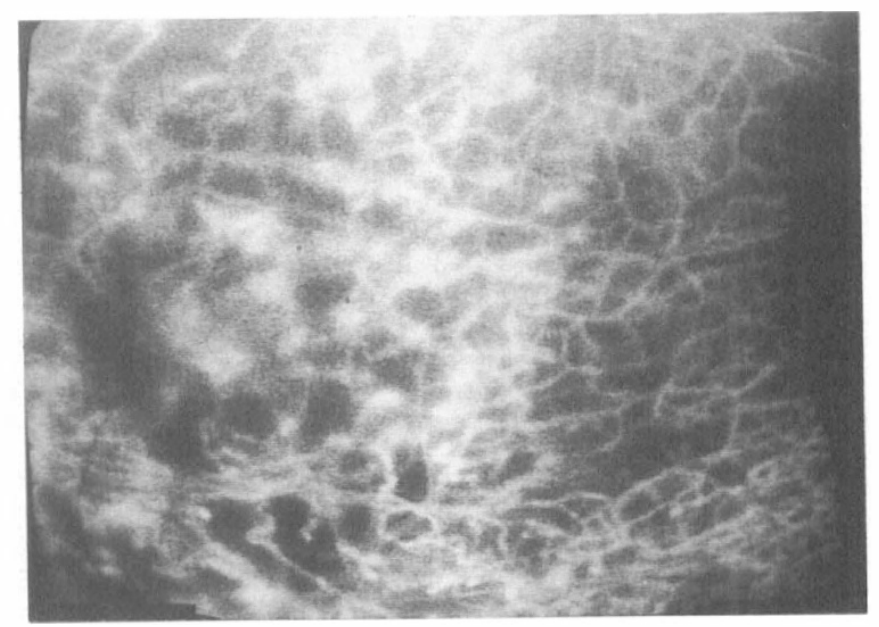

Fig. 7. Early venous phase: lateral episclera. Limbus is at right of photograph. Note apparent absence of 'watershed' zone between territories of anterior ciliary and posterior episcleral vasculatures. Real-time video analysis convincingly demonstrates this to be the case.

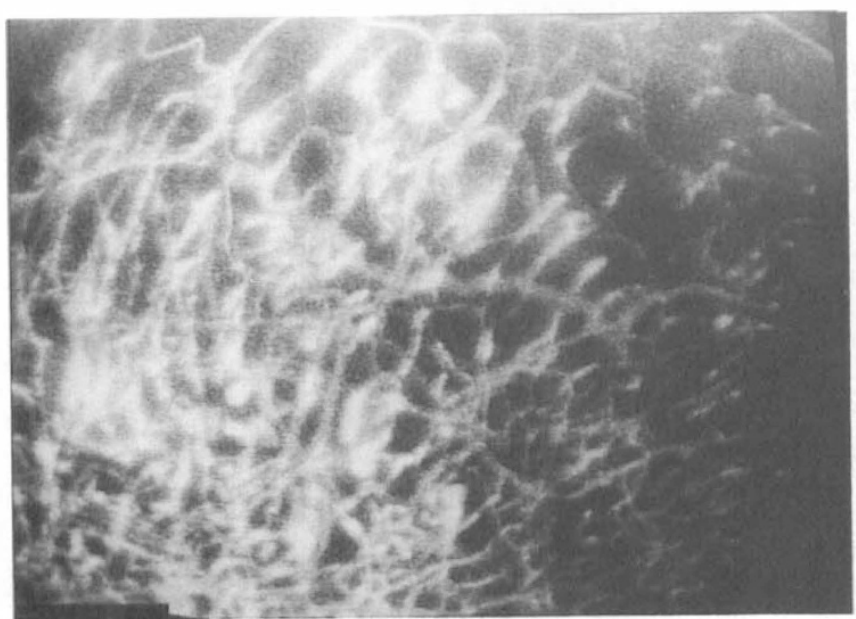

(a)

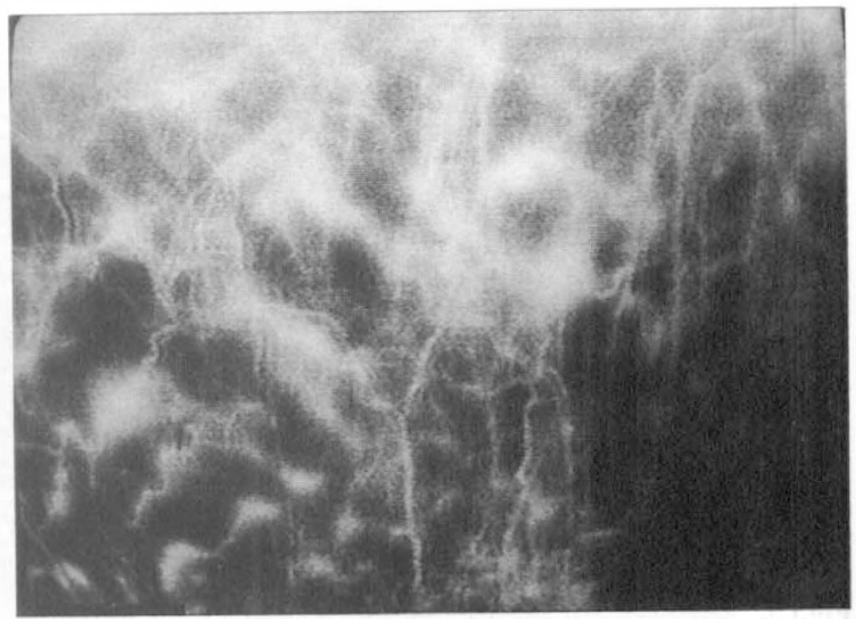

(b)

Fig. 8. Midvenous phase: (a) Temporal episclera. Limbus is at right of photograph. (b) Superior episclera. Limbus is at lower edge of photograph. Note characteristic discontinuous and circumferential dispostion of the 'leaky' vessels and the relative absence of 'leaky' vessels in circumlimbal and forniceal areas.

\section{Flow Kinetics in the Anterior Ciliary Arteries}

A centripetal direction of flow was shown throughout the extent of each anterior ciliary artery, to the termination of their branches as perforating scleral arteries or as ramifications in the anterior episcleral circle. Retrograde (centrifugal) flow was not seen during the fluorescein transit in any of the 37 perforating scleral arteries detected in the 6 subjects. The first few anterograde arterial pulsations of the fluorescein dye front in the anterior ciliary arteries and its primary branches often could be seen. Owing to the rapidity of events, it was not possible to transfer the clear evidence for ciliary artery anterograde flow from the video record into static photographic images made directly from the television monitor.

\section{Arterial Phase}

The lid margin fluoresced 1 or 2 seconds before the dye front was detected in the episcleral circulation. The number of anterior ciliary arteries emergent from the rectus muscle in each quadrant is shown in Table II. Arteries generally filled simultaneously in any one quadrant, but on three occasions (twice in the same individual) there was an approximately 2second filling delay between adjacent anterior ciliary arteries in a single quadrant.

Main anterior ciliary arterial branches approach to within $2-3 \mathrm{~mm}$ of the limbus before ramifying with adjacent vessels. Most of the blood supply was provided by the seven to ten anterior ciliary arteries, although there were occasional components provided by branches from the posterior conjunctival arteries (Fig. 2). An irregular and highly variable 
Table II. Number of anterior ciliary arteries detected by SAM videoangiography

\begin{tabular}{cccccc}
\hline & \multicolumn{3}{c}{ Episcleral quadrant } & \\
\cline { 2 - 5 } Subject & Temporal & Nasal & Inferior & Superior & Total \\
\hline 1 & 1 & 1 & 2 & 3 & 7 \\
2 & 2 & 2 & 3 & 3 & 10 \\
3 & 2 & 2 & 3 & 2 & 9 \\
4 & 1 & 1 & 4 & 2 & 8 \\
5 & 1 & 2 & 2 & 3 & 8 \\
6 & 1 & 2 & 3 & 3 & 9 \\
Total & 8 & 10 & 17 & 16 & \\
\hline
\end{tabular}

anterior episcleral arterial circle was highlighted over a period of several seconds, often with prominent arteriovenous anastomoses (Fig. 3). There was marked variation in the calibre of individual anterior ciliary arteries, which were generally smaller in the horizontal meridia (especially in the temporal quadrant) and larger, multiple, and with more extensive connections in the vertical quadrants (particularly inferiorly). The vertical arteries characteristically supplied large temporal and nasal branches to the episcleral circle (Fig. 4). There was marked individual variability. The perforating scleral arteries were larger vessels than the episcleral branches and passed through scleral foramina, commonly within 1-2 mm of the limbus (Table III).

Over several seconds, an extensive multilayered anterior circumepiscleral plexus was illuminated (Figs. 4, 5). The arterial phase merged imperceptibly with the arteriovenous phase. Flow directions in the episcleral arterial circle were highly variable, and small segments were occasionally seen with temporary cessation of flow. Radial arterioles supplied the limbal arcade (Fig. 6a), and further branches could sometimes be imaged coursing into the limbal palisades of Vogt (Fig. 6b). Recurrent anterior conjunctival arterioles arose from the arcade and passed backwards over the anterior episclera in the limbal reflection of the conjunctiva (Figs. 5, 6).

The posterior conjunctival arteries began to fill 2-8 seconds after the anterior ciliary artery dye front. The episcleral circulation posterior to the rectus muscle insertions was supplied by small branches of the posterior tarsal vessels that passed anteriorly to ramify with the anterior episcleral circulation. In most eyes, although the posterior episcleral circulation was delayed, there was full anastomosis between the two (variable) vascular territories and no evidence for a distinct 'watershed' zone (Fig. 7).

\section{Arteriovenous and Venous Phases}

Filling of the posterior episcleral circulation was usually delayed until the anterior episcleral arteriovenous phase was well under way. Arteries and veins often ran close together and could be differentiated only by careful observation over a period; small
Table III. Number of perforating scleral arteries arising from anterior ciliary arteries or their branches as detected by SAM videoangiography

\begin{tabular}{cccccc}
\hline & \multicolumn{4}{c}{ Episcleral quadrant } & \\
\cline { 2 - 5 } Subject & Temporal & Nasal & Inferior & Superior & Total \\
\hline 1 & 1 & 1 & 2 & 1 & 5 \\
2 & 2 & 0 & 3 & 3 & 8 \\
3 & 1 & 1 & 2 & 2 & 6 \\
4 & 2 & 1 & 2 & 2 & 7 \\
5 & 1 & 1 & 3 & 2 & 7 \\
6 & 0 & 3 & 0 & 2 & 5 \\
Total & 7 & 7 & 12 & 12 & \\
\hline
\end{tabular}

changes in shape and outline associated with changing directions of flow often were seen as the arterial fluorescence was replaced by venous drainage in adjacent vessels. An extensive episcleral vascular net was filled over a few seconds. Directions of flow within this system were complex and polydirectional. Anteriorly, flow in interdigitating channels was principally in an axial direction. Towards the fornices, the net was wider and flow was mainly circumferential, towards the rectus muscle. Overlying conjunctival vessels had to be differentiated from the episcleral plexus; subject blinking was useful to move the conjunctival vessels over the episclera. Aqueous vein laminar flow was not detected using this technique because of the black background produced by tight filtering.

The venous phase was defined by the emptying of the anterior ciliary arteries. The venous drainage of the episclera was complex and multilayered. The superficial episcleral veins draining the limbus were often linear and radial for the first 2-3 $\mathrm{mm}$. Fluorescein leakage occurred early and largely from the episcleral venules; initially, the leakage had a patchy distribution and appeared to occur more prominently from circumferential vessels (Fig. 8 ). The leakage of fluorescein was uncommon in the conjunctival and perilimbal vessels of most individuals. Leakage was notably greater in the interpalpebral area. In the late phase, the sub-Tenon's space was filled diffusely with fluorescein that obscured the episcleral vasculature and highlighted the empty conjunctival vessels.

\section{DISCUSSION}

The SAM provides a solution to several problems associated with anterior segment fluorescence angiography. Its even, coaxial illumination, large depth of focus, real-time video recording, and fast optical speed provide advantages that are not available to an appreciable extent with close-up photographic methods or with modern videocamera techniques. ${ }^{26}$ The difficulties associated with intravascular fluorescence induction across a curved reflecting surface 
when using uneven, off-axis illumination have largely been overlooked.

An important problem with the static presentation of intermittently sampled data that are obtained with conventional anterior segment angiography is the proper identification of arteries and veins. ${ }^{22}$ This is a manifestation of both the structural complexity of the vascular bed and the rapidity of events in the episcleral-conjunctival circulation. However, interpretation of the fluorescein transit in optimised, dynamic SAM videoangiography makes their distinction very clear as origins, sequence, flow and interconnections are clearly observed. For example, it is claimed that because the colour of the episcleral vessels is an unreliable indicator of whether an anterior ciliary vessel is an artery or vein, morphological features alone are the most reliable criteria, such that small, straight scleral vessels are probably veins and wide, tortuous vessels are probably arteries. $^{22}$ Our dynamic studies do not support this contention, because arterial width varies markedly, and we suspect that tortuosity is a manifestation of hypertensive, diabetic or age-related changes, ${ }^{31,32}$ not seen in our relatively young cohort. SAM videoangiography can resolve such interpretive difficulties.

Our data strongly support the contention that the angiographic evidence for primarily retrograde flow in the anterior ciliary arteries from an intracular source $^{17-25}$ is mistaken and explained on methodolgical grounds. We investigated the vascular kinetics of each of the anterior ciliary arteries and their perforating scleral branches in 6 subjects by SAM fluorescein angiography and failed to find vessels that did not have centripetal or anterograde flow. We believe that there are technical reasons why conventional angiographic techniques have usually, ${ }^{17-25}$ but not always, ${ }^{31,33}$ led to the contrary conclusion, and these have been discussed above. All four quadrants of 6 healthy subjects with ages ranging between 21 and 42 years were investigated by strict protocol. Although a small sample size, the previous investigation of 11 individuals ${ }^{26}$ showed identical flow patterns in all the anterior ciliary arteries studied.

Some studies reporting retrograde flow have investigated mainly elderly patients. ${ }^{22,34}$ Although the possibility may exist that anterior ciliary artery flow may become reversed with advancing age, we know of no supportive data. Furthermore, Laatikainen ${ }^{31}$ reported evidence for centripetal flow in 156 eyes of an elderly population with a high prevalence of glaucoma. In addition, the potential implications for fluorescence angiography of the thicker, more opaque Tenon's fascia near the extraocular muscles ${ }^{3}$ and the thinner fascia close to the limbus ${ }^{3}$ have been overlooked. It needs to be considered whether the anterior ciliary arteries might sometimes be compressed within the rectus muscle sheaths at extreme positions of gaze, with consequent reversal of their flow, but we find no evidence that this occurs. The anatomical and clinical evidence supporting this centripetal (anterograde) direction of flow in the anterior ciliary arteries is substantial. ${ }^{1-16}$

The best argument supporting the validity of the directional interpretations of blood flow in SAM videoangiography is the internal consistency provided by the appropriateness of the direction and rate in vessels of different configurations throughout the vascular transit in all quadrants. It is believed that the spots of low fluorescence represent erythrocyte rouleaux formations (as red cells absorb fluorescein poorly) and that the highly fluorescent spots are due to segments of cell-free plasma. ${ }^{35}$ Fluorescence induction in blood is essentially a surface phenomenon, ${ }^{36}$ and rouleaux may tumble into and out of the surface layer. There is no 'particulate' motion in areas of perivascular fluorescein leakage. Flow direction can be discerned in virtually every episcleral vessel that can be imaged, except where overlapping occurs.

The vascular organisation revealed by this study is similar to that shown by montage reconstruction of methylmethacrylate corrosion castings of the nonhuman primate eye. ${ }^{8}$ The anterior episcleral circle is less well developed and more irregular in man. However, the much larger vertical anterior ciliary arteries are a shared feature, and these vessels generally give major medial and lateral branches to the arterial circle. The numbers and distribution of anterior ciliary perforating arteries were in general agreement with a recent biomicroscopic study, ${ }^{37}$ they were almost twice as common in the vertical compared with the horizontal meridia. Scleral emissary vessels were small and seen in only 3 of 12 horizontal and in no vertical quadrants. Occasionally, small posterior conjunctival arteries contributed directly to the anterior episcleral circle, ${ }^{31}$ which was otherwise derived directly from branches of the anterior cilary arteries. The antecubital-episcleral fluorescein perfusion time $^{31}$ was greater than the reported antecubital-retinal transit times.

Our findings of vascular organisation of the anterior episcleral and limbal circulations were in general agreement with the detailed descriptions of Meyer and colleagues, ${ }^{24,25,38}$ except that we were unable to find cogent evidence for the existence of discrete vascular fields (in the sense of arteriovenous units) and, in most eyes, for a 'watershed' zone between the anterior and posterior episcleral circulations. The pattern was one of extensive collateral flow between regions, ${ }^{8}$ as is evidenced by clinical recovery following extraocular muscle tenotomies. We also observed that arterial-arterial ${ }^{24,25}$ 
and arteriovenous ${ }^{25,39-41}$ anastomoses were common in the episcleral vasculature. Despite vasodilation from the use of topical proparacaine in superior quadrant angiograms, necessitated by the lid speculum, no dynamic differences could be discerned in the vascular dynamics of this segment compared with the others.

The majority of episcleral vessel are reported to be venules. ${ }^{42}$ Fluorescein leakage was patchy and occurred around small segments of vessels. Leakage was greater in the horizontal than in the vertical meridia and was most extensive in the region where the anterior and posterior episcleral circulations abutted; leakage was less from perilimbal and conjunctival venules. ${ }^{34}$ The precise nature and significance of these 'leaky' venous segments await further study. Under the standard conditions of this investigation, episcleral fluorescein leakage varied significantly between individuals, but was uniform within the individual. The flow patterns within the microcirculation were decidedly multidirectional as has been described previously using red-free videomicroscopy. ${ }^{38}$

Key words: Anterior ciliary artery, Anterior episcleral vasculature, Blood flow, Episcleral fluorescein angiography, Scanning angiographic microscope.

\section{REFERENCES}

1. Leber T. Die Circulation und Ernahrungsverhaltnisse des Auges. In: Saemisch T, editor. Graefe Saemisch Handbuch der Gesamten Augenheilkunde, 2nd ed, vol 2. Leipzig: Wilhelm Engelmann, 1903:1-89, 197-293.

2. Salzmann M. The anatomy and histology of the human eyeball in the normal state: its development and senescence. Brown EVL, translator. Chicago: Franz Deuticke, 1912:21-2.

3. Duke-Elder S, Wybar KC. System of ophthalmology. Vol II. The anatomy of the visual system. St Louis: Mosby, 1961:347-56, 451-3.

4. McKeown CA, Lambert HM, Shore JW. Preservation of the anterior ciliary vessels during extraocular muscle surgery. Ophthalmology 1989;96:498-507.

5. Ashton N, Smith R. Anatomical study of Schlemm's canal and aqueous veins by means of Neoprene casts. III. Arterial relations of Schlemm's canal. $\mathrm{Br}$ J Ophthalmol 1953;37:577-86.

6. Shimizu K, Ujiie K. Structure of ocular vessels. New York: Igaku-Shoin, 1978:125-9.

7. Woodlieff NF. Initial observations on the ocular microcirculation in man. I. The anterior segment and extraocular muscles. Arch Ophthalmol 1980;98: 1268-72.

8. Morrison JC, Van Buskirk EM. Anterior collateral circulation in the primate eye. Ophthalmology 1983;90:707-15.

9. Hiatt RL. Production of anterior segment ischemia. Trans Am Ophthalmol Soc 1977;75:87-102.

10. Hayreh SS, Scott WE. Fluorescein iris angiography. II. Disturbances in iris circulation following strabismus operation on the various recti. Arch Ophthalmol 1978;96:1390-400.

11. Olver JM, Lee JP. The effects of strabismus surgery on anterior segment circulation. Eye 1989;3:318-26.
12. Wilcox LM, Keough EM, Connolly RJ, Hotte CE. The contribution of blood flow by the anterior ciliary arteries to the anterior segment in the primate eye. Exp Eye Res 1980;30:167-74.

13. Wilcox LM, Keough EM, Connolly RJ. Regional ischemia and compensatory vascular dynamics following selective tenotomy in primates. Exp Eye Res 1981;33:353-60.

14. Leinfelder PJ, Black NM. Experimental transposition of the extraocular muscles in monkeys: a preliminary report. Am J Ophthalmol 1941;24:1115-20.

15. Girard LJ, Beltranena F. Early and late complications of extensive muscle surgery. Arch Ophthalmol 1960;64:576-84.

16. Virdi PS, Hayreh SS. Anterior segment ischemia after recession of various recti: an experimental study. Ophthalmology 1987;94:1258-71.

17. Bron AJ, Easty DL. Fluorescein angiography of the globe and anterior segment. Trans Ophthalmol Soc UK 1970;90:339-67.

18. Amalric P, Rebière P, Jourdes JC. Nouvelles indications de l'angiographie fluorescéinique du segment antérieur de l'oeil. Ann Oculist 1971;204:455-68.

19. Deodati F, Bec P, Labro J-B. Angiographie fluorescéinique du segment antérieur de l'oeil. Arch Ophtalmol 1971;31:768-80.

20. Ikegami M. Fluorescein angiography of the anterior ocular segment. I. Hemodynamics in the anterior ciliary vessels. Acta Soc Ophthalmol Jpn 1974;78:371-85.

21. Kottow MH. Anterior segment fluorescein angiography. Baltimore: Williams and Wilkins, 1978:35-55.

22. Talusan ED, Schwartz B. Fluorescein angiography: demonstration of flow pattern of anterior ciliary arteries. Arch Ophthalmol 1981;99:1074-80.

23. Watson PG, Bovey E. Anterior segment fluorescein angiography in the diagnosis of scleral inflammation. Ophthalmology 1985;92:1-11.

24. Meyer PAR, Watson PG. Low dose fluorescein angiography of the conjunctiva and episclera. $\mathrm{Br} \mathrm{J}$ Ophthalmol 1987;71:2-10.

25. Meyer PAR. Patterns of blood flow in episcleral vessels studied by low-dose fluorescein videoangiography. Eye 1988;2:533-46.

26. Ormerod LD, Fariza, E, Hughes GW, Doane MG, Webb RH. Anterior segment fluorescein videoangiography with a scanning angiographic microscope. Ophthalmology 1990;97:745-51.

27. Webb RH, Hughes GW. Scanning laser ophthalmoscope. IEEE Trans Biomed Eng 1981;28:488-92.

28. Webb RH, Hughes GW, Delori FC. Confocal scanning laser ophthalmoscope. Appl Opt 1987;26:1492-9.

29. Mainster MA, Timberlake GT, Webb RH, Hughes GW. Scanning laser ophthalmoscopy: clinical applications. Ophthalmology 1982;89:852-7.

30. Flower RW. Injection technique for indocyanine green and sodium fluorescein dye angiography of the eye. Invest Ophthalmol 1973;12:881-95.

31. Laatikainen L. Fluorescein angiographic studies of the peripapillary and perilimbal regions in simple, capsular and low-tension glaucoma. Acta Ophthalmol (Copenh) 1971;111(Suppl):54-73.

32. Siprova H, Vacek L. Alternsveranderungen der konjunktivalen Blutgefasse. $\mathrm{Z}$ Alternsforsch 1985;40:51-5.

33. Brancato R, Frosini R, Boschi M. L'angiografia superficiale a fluorescein del bulbo oculare. Ann Ottalmol Clin Ocul 1969;95:433-9.

34. Ikegami M, Maruyama A. Fluorescein angiography of 
the anterior ocular segment. II. Permeability of conjunctival vessels in normal and diseased states. Acta Soc Ophthalmol Jpn 1975;79:1393-404.

35. Wolf S, Arend O, Toonen H, Bertram B, Jung F, Reim M. Retinal capillary blood flow measurement with a scanning laser ophthalmoscope: preliminary results. Ophthalmology 1991;98:996-1000.

36. Delori FC, Castany MA, Webb RH. Fluorescence characteristics of sodium fluorescein in plasma and whole blood. Exp Eye Res 1978;27:417-25.

37. Norn M. Topography of scleral emissaries and scleraperforating blood vessels. Acta Ophthalmol (Copenh) 1985;63:320-2.

38. Meyer PAR. The circulation of the human limbus. Eye 1989;3:121-7.
39. Lee R, Holze EA. The peripheral vascular system in the bulbar conjunctiva of young normotensive adults at rest. J Clin Invest 1950;29:146-50.

40. Gaasterland DE, Jocson VL, Sears ML. Channels of aqueous outflow and related blood vessels. III. Episcleral arteriovenous anastomoses in the rhesus monkey eye (Macaca mulatta). Arch Ophthalmol 1970;84:770-5.

41. Casali AM, Lambertini F, Cavalli G. Flow regulating structures in vessels of the human conjunctiva and eyelid. Boll Soc Ital Biol Sper 1975;51:1903-7.

42. Raviola G. Conjunctival and episcleral blood vessels are permeable to blood-borne horseradish peroxidase. Invest Ophthalmol Vis Sci 1983;24:725-36. 